\title{
Method of Differential Chronoscopy of Unstable Regimes of Combustion Wave Distribution
}

\section{G. Bebiya, M. P. Boronenko, P. Yu. Gulyaev, and P. A. Yurukin}

Yugra State University, Khanty-Mansiysk, Russia

\section{Abstract}

In this paper, mathematical methods for processing a video signal of a high-speed television measuring system are considered. They allow us to determine the unstable combustion regimes in the technology of self-propagating high-temperature synthesis (SHS). The registration of the propagation of the SAF combustion wave was carried out using an optoelectronic micro-pyrometry complex of nanosecond resolution based on the super high-speed television camera "Video SprintNG". The result of

Corresponding Author:

A. G. Bebiya

bebiya.nastya@mail.ru

Received: 21 December 2017

Accepted: 15 April 2018

Published: 6 May 2018

Publishing services provided by Knowledge $\mathrm{E}$

(c) G. Bebiya et al. This article is distributed under the terms of the Creative Commons

Attribution License, which permits unrestricted use and redistribution provided that the original author and source are credited.

Selection and Peer-review under the responsibility of the MIE-2017 Conference Committee.

\section{G OPEN ACCESS} the $2 \mathrm{D}$ visualization of the heat transfer velocity of the SHS wave was obtained by interpreting the difference in the coordinates of the wave front along the entire series of thermal imaging video frames, and indicates that the structure of the DCS is more ordered in time and allows one to judge the spatial and temporal "coherence" of the SHS wave. To detect the transient modes of SHS, the method of pattern recognition based on the Trace transform was used.

Keywords: high-speed television recording, differential chronoscopy, trace transform, SHS combustion wave.

\section{INTRODUCTION}

At present, its important applications in the development of self-propagating hightemperature synthesis (SHS) include its application in the field of additive technologies, for the development of $3 \mathrm{D}$ printers of finished products from metal and metal ceramics [1]. The main controlled parameters are the temperature and propagation velocity of the SHS combustion wave from a molded powder mixture of the reaction starting materials [2]. As a result of the influence of the random structure of the powder mixture of the initial products, the combustion wave front quasiperiodically changes temperature, velocity, and propagation direction [3], which necessitates the development of means for analyzing local instability of the motion of the SHS combustion wave. Traditionally, in order to track the random trajectory of the object's motion, television-measuring systems based on dissectors were successfully used [4], 
the logical development of which were high-speed streak cameras of nanosecond resolution, used to study fast burning processes [5] and propagation of shock waves [6].The experience of using national television measuring systems of nanosecond resolution $[7,8]$ for high-speed recording of SHS processes has shown that they have high metrological characteristics $[9,10]$, necessary for controlling the speed and temperature of the combustion wave $[11,12]$. At the same time, the practical application of television-measuring systems based on streak cameras is difficult, because of the impossibility of real-time processing of a high-speed video data stream of large volume. The characteristic time scales of the formation of the additive layer in the SHS wave are such that for the quasiperiodical cyclicity of the appearance of an elementary combustion site with a period of 10 to $100 \mathrm{~ms}$ [13], the exothermic burning reaction time does not exceed $1 \mathrm{~ms}$ [14], and the effective times of heat generation and phase formation do not exceed 50-100 microseconds [15]. Thus, any reduction in the frame frequency or spatial thinning of the frame leads to loss of control over the combustion transition to the critical regime, if the registration frequency does not exceed by one order of magnitude the characteristic scale of the local instability effects [16]. This task of analyzing the microheterogeneous combustion structure is called the SHS discreteness problem, and in most cases is solved experimentally because the conclusions of different theoretical models on the behavior of the combustion wave at a macroscopic or microscopic level contradict each other [17]. Ways to solve this problem, the authors see in the application of mathematical methods of video data compression, by introducing the interframe difference into the signal processing path, and parametrization of the results of differential chronoscopy using algorithms such as Fast Fourier Transform (FFT), Trace-Transform (TT) or Hafa algorithms [18].

The aim of the work is to select a feature for recognizing the critical modes of propagation of the SHS combustion wave from high-speed video data. To do this, we must solve three problems: to offer an efficient algorithm for reducing the redundancy of the video stream based on the interframe difference; select the most sensitive to the change in the technological mode of combustion, the core of the Trace-conversion; to offer statistical features for the recognition of the critical combustion regime of SHS.

\section{TECHNIQUE AND TECHNIQUE OF THE EXPERIMENT}

To record the propagation of the SAF wave of combustion, an optico-electronic micropirometry complex of nanosecond resolution was used on the basis of a super high-speed television camera "VideoSprintNG" (CJSC NPK Vidiscan, Russia) with an electron-optical converter-photomultiplier on a microchannel plate and an optical 
shutter "Nano-Gate". TV measuring complexes based on high-speed CMOS photomatrix using functional modes of multi-exposure and "global shutter" [9] in the channel of optical-electronic conversion greatly expand the possibilities of studying fast combustion processes of SHS, due to multiple recording of the image of a moving object with nanosecond delays between frames [10]. The combustion wave was investigated in a well-studied $\mathrm{Ni}$-Al system of equimolar composition [19], with inert additives of the final reaction product in a weight ratio of o to $30 \%$ in $2.5 \%$ increments. This allowed for a controlled transition to unstable combustion regimes.
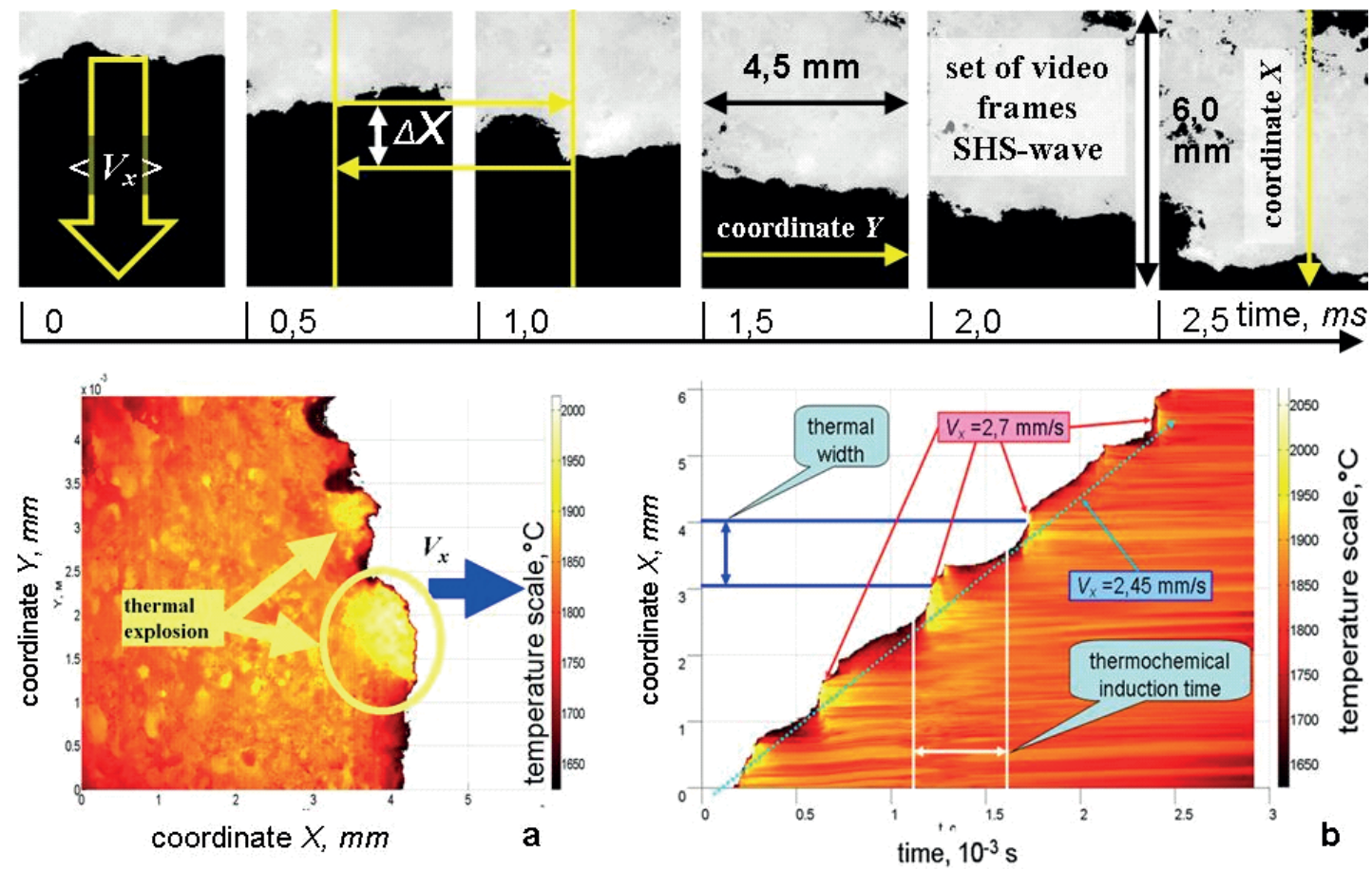

Figure 1: Video frames of the SHS wave: a - temperature field $T(x, y)$ in one frame; $b$ - temperature chronogram $T(x, t)$ and wave front motion $X=f(t)$ along one line.

Figure 1 shows an example of high-speed micro-video shooting, and, in Fig. 1 (a), one frame with the temperature field $T(x, y)$ of the combustion wave at time $t$ is given, and in Fig. 1 (b) the principle of measuring the average wave velocity burning $\langle\mathrm{Vx}\rangle$ along the selected row with the $Y$ coordinate [11]. High spatial resolution (1200x800 pxl), short multi-exposure time (3ons) and interframe interval ( $\Delta \mathrm{t} \leq 1 \mathrm{~ms})$ provide detailed visualization of the fine thermal structure of the SHS combustion wave [12].

\section{COMPUTER MODELLING}

The video stream of the data of the high-speed video camera "Video SprintNG" is controlled by the library functions of the specialized image program Image) (National 
Institutes of Health, USA) with open source software designed for analysis and processing of scientific multidimensional images. o compress data, the authors used the method of differential chronoscopy (DXS), which is a modified algorithm for calculating the interframe difference [13]. According to the temperature chronogram $T(x, t)$ shown in Fig. 1 (b), the threshold for the binarization of the image of the temperature field in the combustion wave of SHS was determined in such a way that the boundary of the wave front was reliably identified. In our case, the threshold value was chosen equal to $1638^{\circ} \mathrm{C}$, which in the phase diagram of the solubility of the $\mathrm{Ni}$-Al system corresponds to the temperature of the peretectic decomposition of the intermetallide Ni1Al1. As a result of threshold binarization of video frames, instead of a stream of video data with a large volume and significant redundancy, for each frame, we obtain the vector of $X$ $(t, y)$ - coordinates of the wavefront of the SHS, where $t$ is the frame time, and $y$ is the line number. The final result of the $2 \mathrm{D}$ visualization of the heat transfer velocity of the SHS wave is obtained by interpreting the coordinate difference $\Delta \mathrm{X}(\mathrm{t}, \mathrm{y})=\mathrm{X}(\mathrm{t}, \mathrm{y})$ $-X(t-\Delta t, y)$ of the wave front along the entire series of thermal imaging video frames and is shown in Figure 2. Obviously, that the structure of the DXS is more ordered in time and allows us to judge the spatial and temporal "coherence" of the SHS wave.

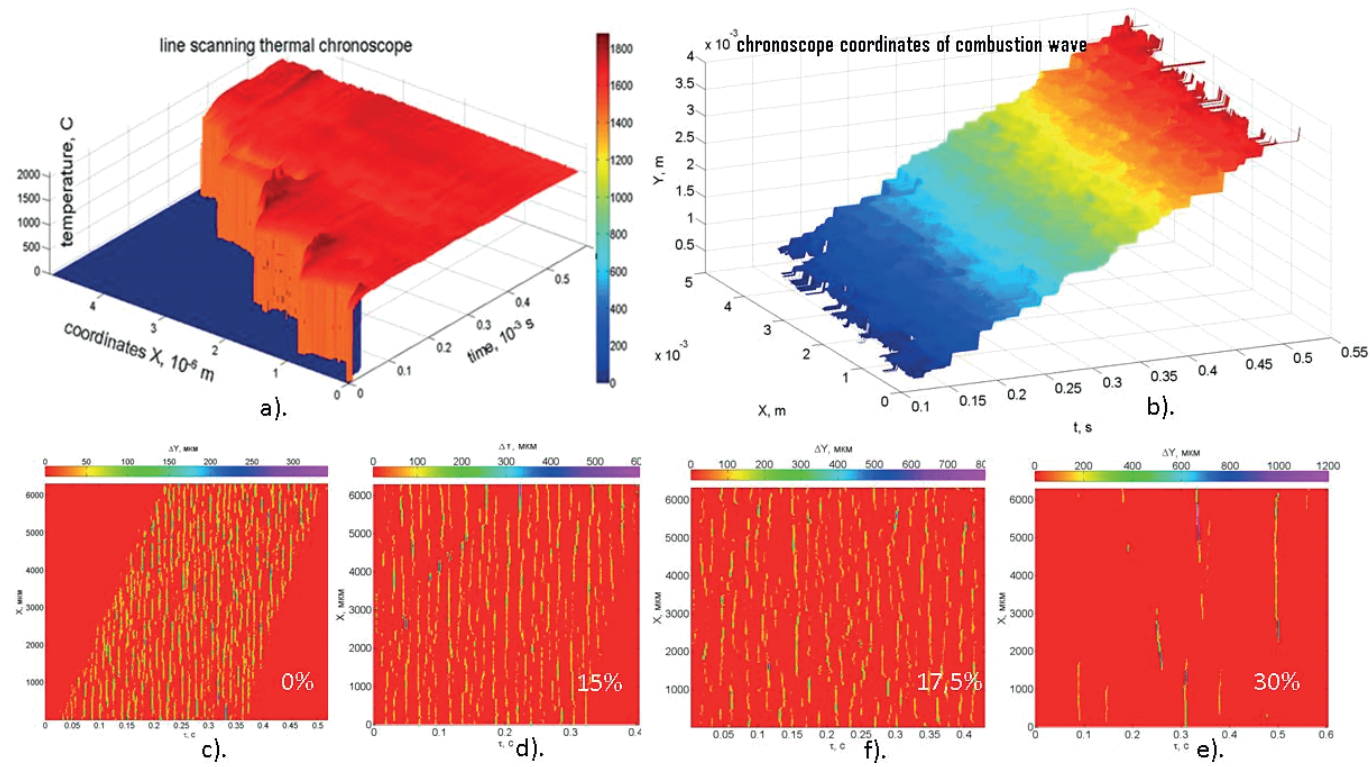

Figure 2: The procedure for obtaining the DCS of the combustion wave: $a-T(x, t)$ lines; $b$ - coordinates of the front $X(t, y)$ in all rows; $c$ - DXC of pure combustion products; $d$ and $f$ - DCS with $15-17 \%$ inertness; e - unstable mode with $30 \%$ inert.

The statement of the computer simulation problem for idealized SHS regimes was based on the space-time structure of the experimental DXS and additional conditions of chemical combustion physics in the form of the Hume-Rothery criterion, according to which the trajectory of the chemical reaction becomes unstable when stoichiometry is 

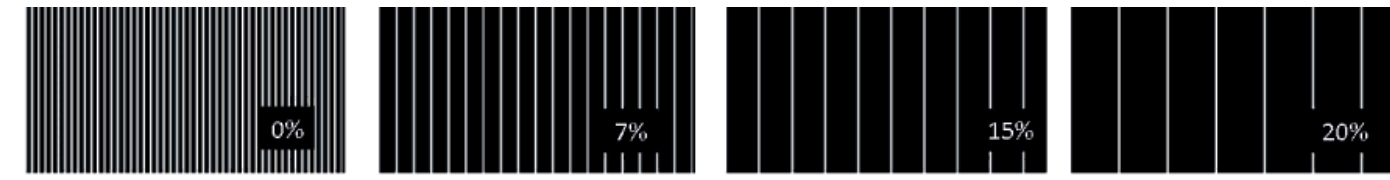

a).
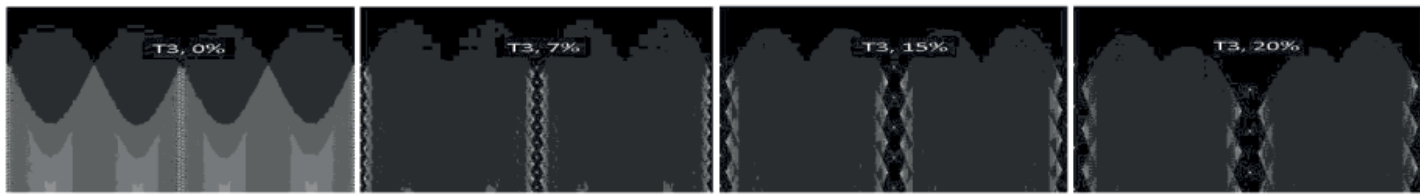

b).
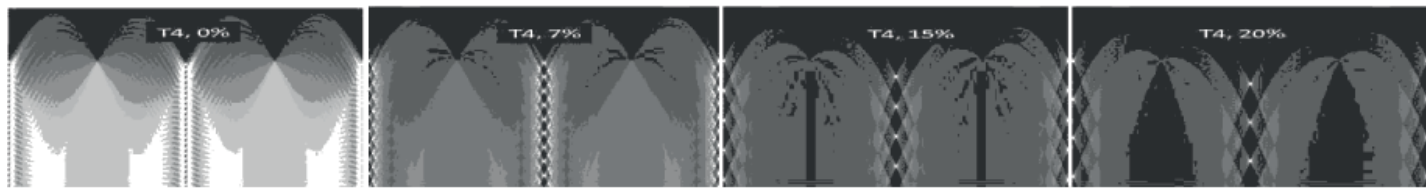

c).

Figure 3: Model of the combustion wave: a- DCS with an inert additive of 0\%, 7\%, 15\%, 20\%; b - Trace images of T3; c - Trace images T4.

violated by more than $15 \%$. In our case, this is confirmed experimentally by introducing impurities in the form of inert or alloying additives [19], as can be seen from Fig. 2 (d) and ( $f$ ). As it was established earlier [3], the discreteness of the SHS thermal structure in this case is directly proportional to the mass fraction of additives in the range up to $12-15 \%$, and the higher the wave decays, the combustion becomes locally unstable and goes into a damped or pulsating relay regime. To identify the transient modes of SHS, the method of pattern recognition based on the Trace transform was chosen [20], which proved itself well in solving analogous problems of determining the invariants of high-speed emissions in arc-arc deposition technologies [21]. The IMS model of the SHS combustion wave, under conditions of dilution of the initial mixture with an inert additive, was calibrated according to the experimental data given above and is shown in Fig. 3, together with examples of their Trace images for typical functionals $T_{3}, T_{4}$ and 55 corresponding to the 3,4 , and 5 harmonics the Radon-Nicodem transformation [20]:

$$
\begin{gathered}
T(f(t))=\left|\int_{c *}^{\infty} e^{i 5 \log (r)} r f(t) d t\right| \\
T(f(t))=\left|\int_{c *}^{\infty} e^{i 3 \log (r)} f(t) d t\right| \\
T(f(t))=\left|\int_{c *}^{\infty} e^{i 4 \log (r)} \sqrt{r} f(t) d t\right|
\end{gathered}
$$

As can be seen in Figure 3, the sensitivity of the recognition depends on the choice of the Trace-functional when the discreteness of the SHS wave changes: for T3, fan 


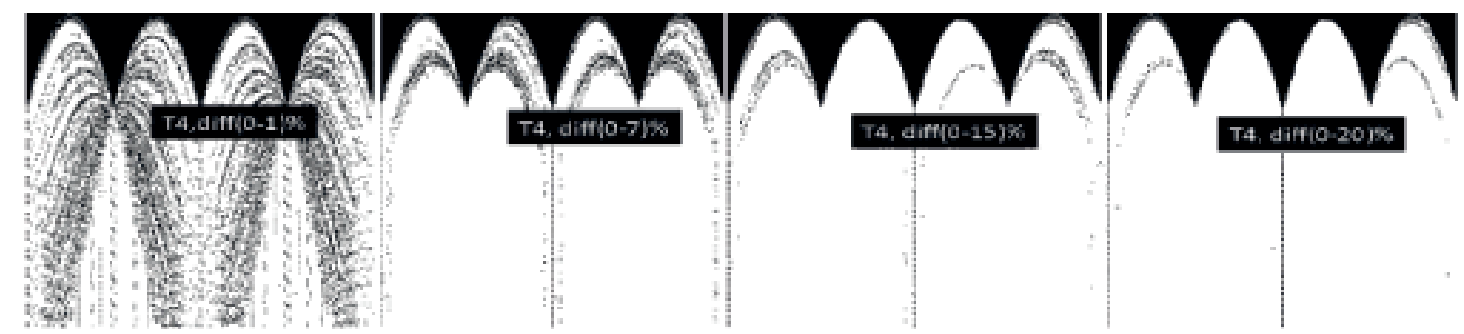

a).

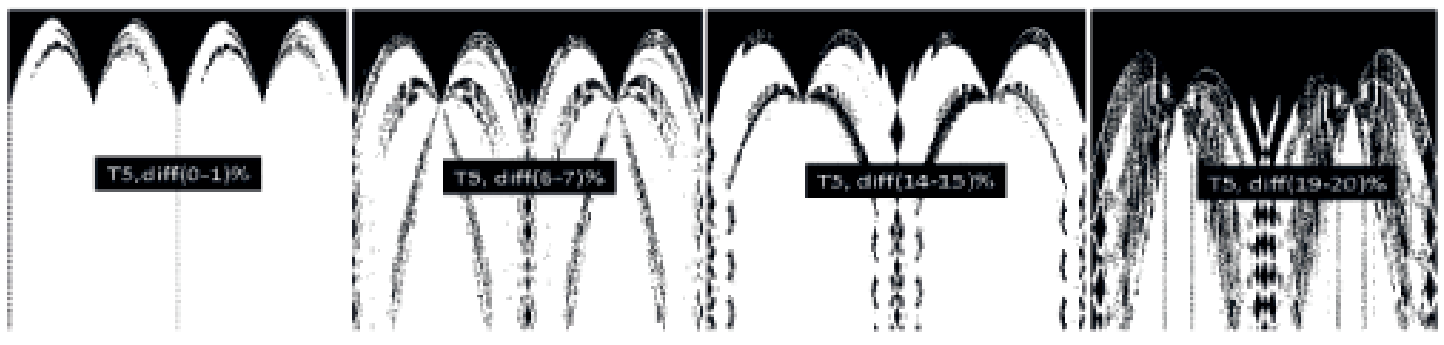

b).

Figure 4: Differential sensitivity analysis: a- "base-analysis" for Trace-images T3; b - «near-analysis» Trace-images T5.

structures are characteristic; T4 recognizes the slope of the wave; T5 monitors the parabolic heat transfer profile. In order to determine the best sensitivity of recognition of the critical combustion regime, when the $15 \%$ dilution was reached with an inertium, the difference in the Trace matrices between the base image (o\% impurity) and the current one - the "base analysis", and the "near-analysis" between adjacent Trace At each step of the additive admixture. The results are shown in Figure 4.

\section{RESULTS AND DISCUSSION}

Visualization of difference Trace matrices, obtained during the application of the algorithms of the "base-analysis" and "near-analysis", makes it possible to conduct a statistical analysis of the behavior of their histories of brightness as the inert additive increases from $0 \%$ to $20 \%$, as shown in Fig.

Obvious signs of the behavior of histograms obtained during the "near-analysis" is a 3 -fold decrease in the distribution amplitude for T4 in Fig. 5 (a) and the shift of the average luminance value for T5 with "base-analysis". The functional T3 should be recognized as the least effective for solving problems of recognition of critical SHS regimes. 


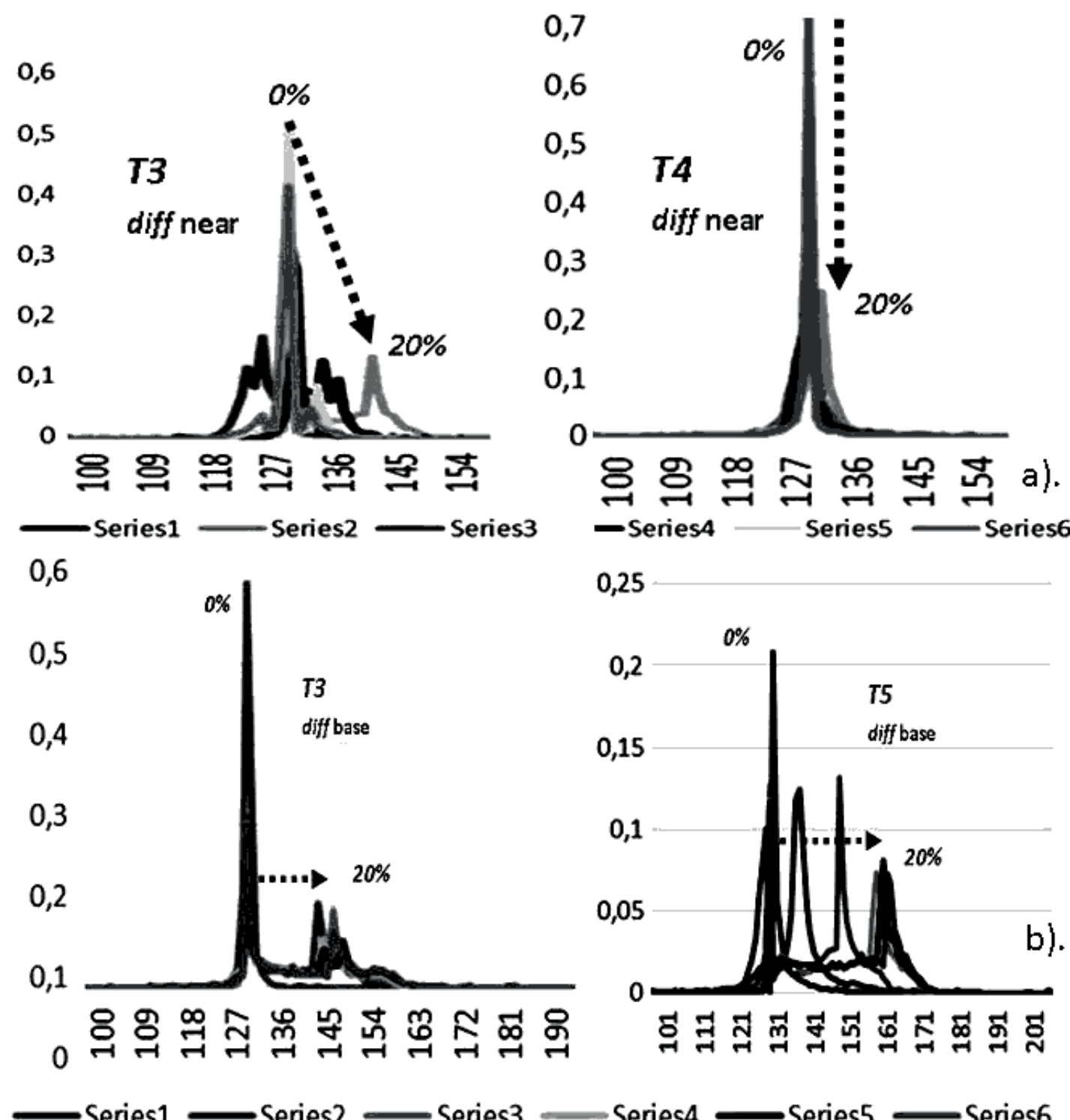

Figure 5: Statistical features of recognition of critical combustion conditions of SHS: a - amplitude for "near-analysis"; b - variance of the "base-analysis".

\section{CONCLUSION}

The revealed differences allow creating a database about Trace-transform invariants corresponding to unstable combustion modes in the SHS process.

Prospects:

- The use of DXS for analysis of the combustion wave makes it possible to introduce the concept of "coherence" of the SHS process.

- Increasing the sensitivity of the Trace analysis opens the possibility of investigating the processes of structurization of SHS in situ. 


\section{ACKNOWLEDGEMENTS}

The work was carried out with the partial financial support of the RFBR within the framework of the scientific project No. 15-42-00106 (SUSU) of the regional program for KhMAO-Ugra.

\section{References}

[1] Evstigneev V.V. Development Prospects of SHS Technologies in Altai State Technical University / V.V. Evstigneev, I.V. Miljukova, V.D. Goncharov, V.A. Vagner, A. A. Gladkih et al. // International Journal of Self-Propagating High-Temperature Synthesis. 2006. - T. 15, No. 1. - P. 99-104.

[2] Evstigneyev V.V. A new procedure of high-rate brightness for SHS processes / V.V. Evstigneyev, A.B. Mukhachev, D.A. Garkol et al. // Combustion, Explosion and Shock Waves. - 1994. - Vol. 30, No. 1. - P. 72-78.

[3] Kalachev A.V. Investigation of the thermal structure of the combustion wave of self-propagating high-temperature synthesis. Kalachev, P.Yu. Gulyaev, V.I. Iordan // News of Altai State University. - 2005. - No. 1 (45). - P. 104-109.

[4] Kazantsev GD Measuring television: a textbook for universities / GD Kazantsev, MI Kuryachii, IN Pustynsky. - Moscow: Bысш. sc., 1994. - 228c.

[5] Nagase K. Spectroscopic Analysis of Diesel Combustion Flame by Means of Streak Camera / K. Nagase, K. Funatsu, I. Haga, Lecture Theses of the 7th International Combustion Engine Symposium, Japan. - 1988. - No. 123.

[6] Shepard, C. L. Measurements of lateral thermal smoothing of $0.53 \mu \mathrm{m}$ laser intensity nonunitormities via shock-wave analysis. C. L. Shepard, P. M. Campbell. Physical Review A. 1989. Vol. 39, No. 3. - P. 1344-1350.

[7] Boronenko M.P. TV measuring system of nanosecond resolution / M.P. Boronenko, P.Yu. Gulyaev // Reports of TUSUR. - 2014. - No. 1 (31). - P. 60-64.

[8] Bebia A.G. Optoelectronic Complex for Micropyrometry of Nanosecond Resolution. Bebia, A.E. Seregin, M.P. Boronenko // Modern Technology and Technologies: Sat. tr. XXI International. scientific-practical. Conf. students, graduate students and young scientists, October 5-9, Tomsk, 2015. - P. 246-248.

[9] Boronenko M.P. Increasing the noise immunity of optical-electronic systems based on video cameras with an optical converter / M.P. Boronenko, A.E. Seregin, K.G. Poluhina et al. // JPCS. - 2015. - Vol. 643. - Article Id 012028. 
[10] Borodina K.A. Per-pixel adjustment of the afterglow effect EOC screen / K.A. Borodina, S.A. Sorokina, A. G. Bebiya, M.P. Boronenko et al. // JPCS. - 2016. - Vol. 741, No. 1. - Article Id 012139.

[11] Boronenko M.P. Increasing accuracy of high temperature and speed processes micropyrometry / M.P. Boronenko, A.E. Seregin, A.G. Bebiya et al. // IOP Conference Series: Materials Science and Engineering. - 2015. - Vol. 93, No. 1. - Article Id 012021.

[12] Boronenko M.P. NiAl combustion systems by the thermal fields visualization method / M.P. Boronenko, A.E. Seregin, I.V. Milyukova et al. // Scientific Visualization. - 2015. - Vol. 7, No. 5. - P. 102.-108.

[13] Cui H.Zh. The Temperature Control in the Combustion Wave SHS / H.Zh. Cui, P.Yu. Gulyaev // Multi-core processors, parallel programming, FPGA, signal processing systems. - 2016. - No. 6. - P. 207-211.

[14] Gulyaev P.Yu. Plasma spraying of protective coatings from ferromagnetic SHSmaterials // International Research Journal. - 2013. - No. 12-1 (19). - P. 74-77.

[15] Gulyaev P.Yu. Temperature measurements for Ni-Al and Ti-Al phase control in SHS Synthesis and plasma spray processes / P.Yu. Gulyaev, I.P. Gulyaev, I.V. Milyukova, H.-Z. Cui // High Temperatures - High Pressures. - 2015. - Vol. 44, No. 2. - P. 83-92.

[16] Iordan V.I. A complex of digital image processing methods for studying the effects of local instability and nonstationarity of the combustion wave of the SHS / VI process. Jordan, P.Yu. Gulyaev, V.V. Evstigneev // Polzunovsky Herald. - 2005. - No. 4-1. - P. $152-170$.

[17] Wright A. The Discrete Regime of Flame propagation in Metal particulate clouds / A. Wright, A.J. Higgins, S. Goroshin // Combustion Science and Technology. - 2016. - Vol. 188, No. 11-12. - P. 2178-2199.

[18] Iordan V.I. Upgrading of the hardware and software of the high-speed intelligent video camera / V.I. Jordan, I.K. Ryabchenko // Bulletin of the Ugra State University. - 2014. - No. 2 (33). - P. 74-80.

[19] Song X.-J. Microstructure and evolution of ( $\mathrm{TiB}_{2}+\mathrm{Al}_{203}$ ) / NiAl composites prepared by self-propagation high-temperature synthesis / X.-J. Song, · H.-Z. Cui, · L.-L. Cao et al. // Transactions of the Nonferrous Metals Society of China. - 2016. - Vol. 26, No. 7. - P. 1878-1884.

[20] Kadyrov A. The Trace Transform and its applications / A. Kadyrov, M. Petrou // IEEE Trans. PAMI. - 2001. - Vol. 23 (8). P. 811-828.

[21] Gulyaev P.Yu. Trace Transform invariants of high-velocity energy emissions from the surface of tungsten microdroplets at breakup in the plasma jet / P.Yu. Gulyaev, V.I. Jordan, I.P. Gulyaev, A. V. Dolmatov // In the book: 17th International Conference 
on Radiation Physics and Chemistry of Condensed Matter Abstracts. - Tomsk: TPU Publishing House, 2016. - P. 85. - 112 pp. 